\title{
MHD Simulation of Dark Filament Eruption in the Quadrupole Source Model
}

\author{
Y. Uchida, S. Hirose, S. Cable, S. Uemura, K. Fujisaki, M. Torii and S. \\ Morita
}

Physics Department, Science University of Tokyo, Shinjuku-ku, Tokyo, Japan

\begin{abstract}
A magnetohydrodynamic simulation in a 2.5D approximation is performed for the quadrupole source model for arcade flares treated years ago by Uchida (1980), and recently supported by the observations from Yohkoh. It is shown that this model can explain several key characteristic features of arcade flares found by Yohkoh, and can avoid some of the paradoxes existing in the "classical model".
\end{abstract}

\section{Introduction}

Recent observations by Yohkoh of the faint pre-event coronal structures of arcade flares and high latitude X-ray arcade formation events have revealed that there exist structures like the connections from the top of the flare cusp back to the photosphere on both sides (Uchida 1996), or "overlapped dual arcades" in the pre-event corona before arcade formations (Uchida et al. 1998), quite unexpected from the "classical model" for solar arcade type flares. The findings about the process of X-ray arcade formation itself (Fujisaki et al. 1998) also suggest that the observations are not compatible with what the "classical model" predicts.

In the present paper, we describe our results of $2.5 \mathrm{D}$ magnetohydrodynamic simulations in the quadrupole magnetic source model (Uchida 1980), and present a new interpretation for the dynamic eruption of dark filaments.

\section{Magnetodynamic Simulations with the Quadrupole Source Model}

Based on the new observational findings presented in our review (Uchida 1998, these proceedings), we assume: (i) a quadrupole array photospheric magnetic source (as in Figure 1) which may represent the bipolar field plus a belt of flux patches transported to the other sides, together with the presence of an overall longitudinal (parallel to the central neutral line) field component as found for arcade formations (Uchida et al. 1998), and (ii) a certain amount of gas (dark filament gas) loaded on the part of the longitudinal field in the region of the neutral sheet, and (iii) the photopheric footpoints are squeezed slowly and nearly homologously toward the central polarity-reversal line to prepare the state with energy stored in the form of magnetic stress (Figure 2).

In our model, the longitudinal field, $B_{\|}$, dominates in the neutral sheet because $B_{\perp}$ is zero there, and this $B_{\|}$has a clear role in separating the oppositely- 


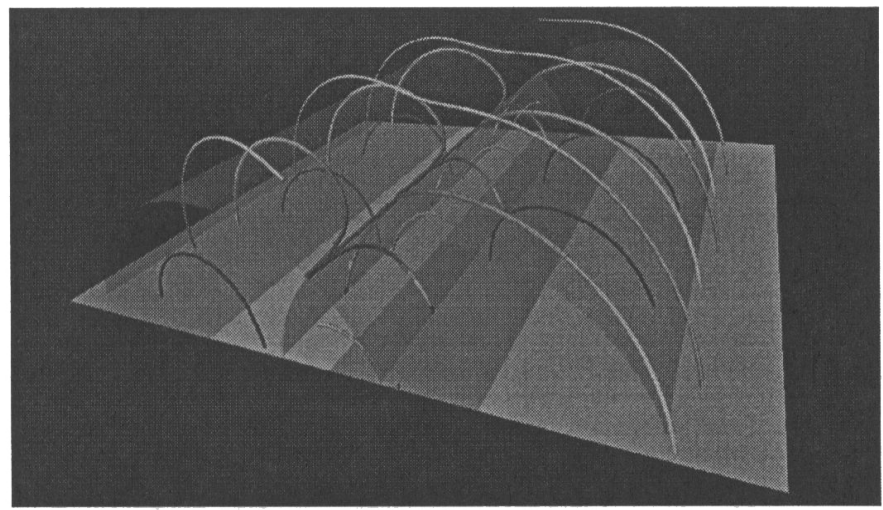

Figure 1. A model magnetic field for quadrupole photospheric sources before squeezing. The separatrix surfaces of "dual arcades" shape is seen, just like the observed "overlapped dual arcades". $B_{\|}$dominates in the region near the "neutral line" (the locus of the neutral points in $B_{\perp}$ ), and the lines of force there are parallel to the neutral line.

directed $B_{\perp}$ on both sides of the thin and vertical neutral sheet in our model, preventing the reconnection. The dark filament mass stabilizes this function. The dark filament here, however, is dynamically passive, and can be squeezed out by external perturbations, and the separation of the opposite-polarity fields $B_{\perp}$ can diminish, allowing the start of reconnection, leading to a reconfiguration by which the stress energy can be relaxed.

The observations by Yohkoh suggest that the region of high latitude dark filaments is very stable for a few solar rotations before destabilization. Therefore, they are likely to be in a relatively low-tension state, rather than something like a highly sheared state. In our model, the elongated form of the dark filament is due to the vanishing $B_{\perp}$ in the neutral sheet, rather than due to a strong shear.

Our interpretation for the destabilization is that there is an injection of current (twist in a small emerging loop may be transferred to our magnetic field system through reconnection) from the emerging flux pair right into the dark filament region. If the injected current has opposite direction to the current in the dark filament, then an expulsive force acts, pushing the upper part of the dark filament upward. At the same time, the lower part is pressed down and heated to make the S-shaped X-ray feature in the dark tunnel.

After the evacuation of the dark filament together with $B_{\|}$, the antiparallel vertical parts of $B_{\perp}$ are pressed into direct contact, and the condition for reconnection is satisfied. Here we use as usual, an anomalous resistivity that enhances the speed of reconnection. (The essential part of the "classical local model for reconnection" is still left unsolved. We come back to this point in a separate paper.) 


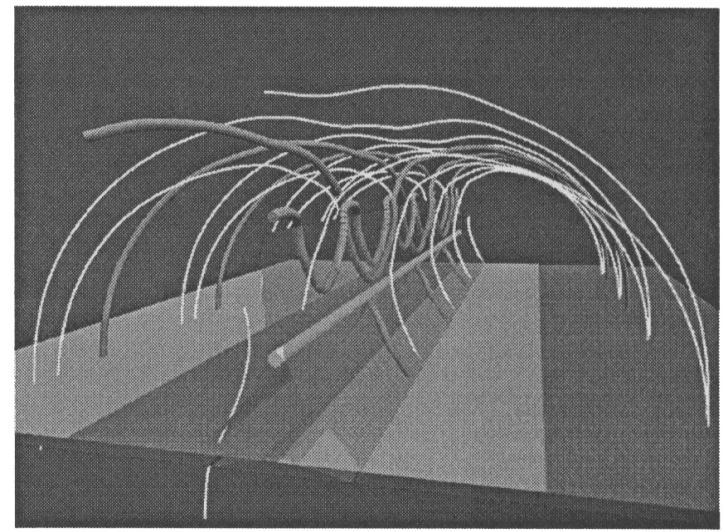

Figure 2. A model magnetic field for quadrupole sources with mass loaded on the field lines near the neutral line, and the footpoints in the photosphere are squeezed nearly homologously toward the central polarity-reversal line (Hirose et al. 1998). (In this particular case, there exists a magnetic island in $B_{\perp}$ in the neutral sheet region which corresponds to a helix in 3D).

\section{MHD Simulation After the Current Injection}

Here, we show some results of 2.5D MHD simulations performed for the abovementioned squeezed quadrupole source model with injection of current as the initial trigger (Hirose et al. 1998) (Figure 3).

It is seen in the first panel (upper left) of Figure 3 that the equilibrium distribution of the dark filament gas held in $B_{\|}$has a thin partition type shape as seen also in Figure 2, separating the oppositely-directed $B_{\perp}$ on both sides. In the second panel (upper right), the upper part of the dark filament is squeezed out, while the lower part is pressed down as the current is injected, providing the model counterparts of erupting dark filament, and of the heated S-shaped structure observed low along the polarity-reversal line (down in the tunnel), respectively. As the reconnection of the main $B_{\perp}$ with the stored stress starts in the part of the neutral sheet from which the dark filament has been squeezed out, the squeezed out dark filament, which "hesitates" to rise, is re-accelerated by the inflating magnetic flux transferred from the squeezed part on both sides into the upper portion through reconnection. The upper part of the dark filament becomes an eruptive dark filament with continued acceleration (lower right). The reconnection produces the main part of the flare, a heated cusped region just like the observed cusps in arcade flares at the limb, but with upper connections connecting the top of the heated cusp back to the photosphere on both sides (Uchida 1996). There is also a feature pressed down (S-shaped structure if seen from above), explaining the observed S-shaped feature inexplicable by the classical model. 


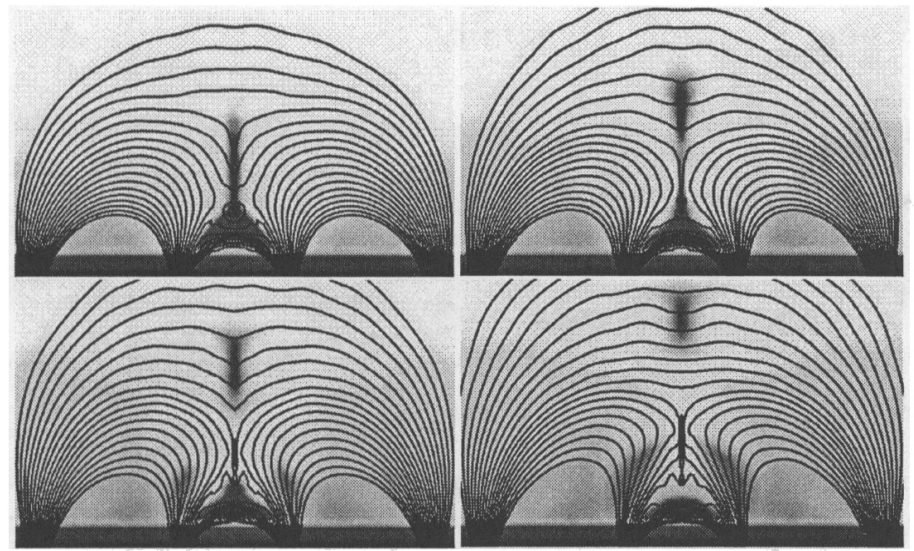

Figure 3. $\quad B_{\perp}$ and density contour projected on the $\mathrm{x}-\mathrm{z}$ plane. Time progresses from upper left, upper right, lower left, and lower right. See the text (Hirose et al. 1998).

\section{Advantages of Quadrupole Magnetic Source Model: Conclusion}

It was shown in the above that the quadrupole source model can explain several key observational features: the pre-event "overlapped dual arcades" type coronal structure as a "pre-heated separatrix surface", and the high bright loops connecting the flare core to both sides as well as the bright feature on the axis of the dark tunnel. Acceleration, rather than deceleration, of the rising dark filament, is also explained.

The quadrupole source model also has merits for avoiding a few, but essential, difficulties in the classical model: (i) the energy paradox in which the dark filament rise should have greater energy than the flare itself, and (ii) the difficulty of supporting the dark filament gas at the top of the otherwise convex arcade (Uchida 1996).

\section{References}

Hirose, S., Cable, S., Uchida, Y. and Uemura, S. 1998, in preparation

Fujisaki, K., Uchida, Y., Morita, S., Hirose, S., and Cable, S. 1998, Publ. Astron. Soc. Japan, submitted

Uchida, Y. 1980, in Solar Flares, (ed.) P.A. Sturrock, University of Colorado Press, Boulder, p. 67 and p. 110

Uchida, Y. 1996, Adv. Space Res., 17, 4/5, 19

Uchida, Y., Fujisaki, K., Morita, S., Torii, M., Hirose, S. and Cable, S. 1998, Publ. Astron. Soc. Japan, submitted 